\title{
Difference in reproductive strategies of two scleractinian corals (branching vs massive) along the west coast of Mexico
}

\section{Diferencia en las estrategias reproductivas de dos corales escleractinios (ramificado vs masivo) a lo largo de la costa occidental de México}

\author{
Héctor Efraín Chávez-Romo ${ }^{1,2}$, David Arturo Paz-García ${ }^{3 *}$, Francisco Correa-Sandoval², \\ Héctor Reyes-Bonilla ${ }^{4}$, Ramón Andrés López-Pérez ${ }^{5}$, Pedro Medina-Rosas ${ }^{6}$ \\ ${ }^{1}$ Facultad de Ciencias Marinas, Universidad Autónoma de Baja California (UABC), Carretera Transpeninsular \\ Ensenada-Tijuana No. 3917, Fraccionamiento Playitas, Ensenada 22860, Baja California, México. \\ 2 Instituto de Investigaciones Oceanológicas, Universidad Autónoma de Baja California, Carretera \\ Transpeninsular Ensenada-Tijuana No. 3917, Fraccionamiento Playitas, Ensenada 22860, Baja California, \\ México. \\ ${ }^{3}$ Laboratorio de Genética para la Conservación, Centro de Investigaciones Biológicas del Noroeste (CIBNOR), \\ Instituto Politécnico Nacional 195, Colonia Playa Palo de Santa Rita Sur, La Paz 23096, Baja California Sur, \\ México. \\ ${ }^{4}$ Departamento de Biología Marina, Universidad Autónoma de Baja California Sur (UABCS), Carretera al Sur \\ Km 5.5, La Paz 23280, Baja California Sur, México. \\ ${ }^{5}$ Instituto de Recursos, Universidad del Mar, Puerto Ángel 70902, Oaxaca, México. \\ ${ }^{6}$ Departamento de Ciencias, Centro Universitario de la Costa-Universidad de Guadalajara, Av. Universidad de \\ Guadalajara No. 203, Delegación Ixtapa, Puerto Vallarta 48280, Jalisco, México.
}

* Corresponding author. E-mail: dpaz@cibnor.mx

\begin{abstract}
This study addressed the relative contribution of sexual and asexual reproduction to the genetic composition of populations of two scleractinian corals, Pocillopora damicornis and Porites panamensis, off the west coast of mainland Mexico. Reproductive indexes showed that $P$. damicornis reproduced both sexually and asexually; $P$. panamensis reproduced sexually, but colonies with an asexual origin were also observed (10-30\%). Asexual reproduction is usually attributed to fragmentation caused by hurricanes; however, no significant association between reproductive index values and frequency of hurricanes was observed for either species. Environmental conditions in the Gulf of California seem to be more favorable for sexual reproduction in both species than other parts of the west coast of Mexico. This study contributes baseline information of differences in sexual and asexual reproduction in massive and branching corals.
\end{abstract}

Key words: sexual reproduction, genotypic diversity, reproductive mode, genetic composition, Eastern Pacific.

RESUMEN. Se estudió la contribución relativa de la reproducción sexual y asexual a la composición genética de dos corales escleractinios, Pocillopora damicornis y Porites panamensis, de la costa occidental de México. Los índices reproductivos mostraron que P. damicornis presentó reproducción sexual y asexual; P. panamensis presentó reproducción sexual, pero también se observaron colonias que tuvieron un origen asexual (10-30\%). La reproducción asexual es usualmente atribuida a la fragmentación por causa de huracanes; sin embargo, no hubo una asociación significativa entre los índices reproductivos de las dos especies y la frecuencia de huracanes. Las condiciones ambientales en el golfo de California parecen ser más favorables para la reproducción sexual en ambas especies que otras partes del Pacífico mexicano. Este estudio contribuye con información básica sobre las diferencias en reproducción sexual y asexual en las especies de coral masivo y ramificado.

Palabras clave: reproducción sexual, diversidad genotípica, modo reproductivo, composición genética, Pacífico oriental.

\section{INTRODUCTION}

Corals are long-lived organisms composed of thousands of individuals in a single colony that can potentially employ different strategies of sexual or asexual reproduction in their life history. Strategies of sexual and asexual reproduction of coral species are important for maintaining resilience of their populations; these strategies represent fundamental evolutionary processes resulting from environmental disturbances of natural or human causes (Harrison 2011). The contribution

\section{INTRODUCCIÓN}

Los corales son organismos longevos compuestos por miles de individuos en una sola colonia que potencialmente pueden emplear diferentes estrategias de reproducción sexual o asexual a lo largo de su historia de vida. Las estrategias de reproducción sexual o asexual de las especies de coral son importantes para mantener la resiliencia de sus poblaciones; estas estrategias representan procesos evolutivos fundamentales que resultan de los disturbios ambientales naturales o 
of sexual and asexual reproductive strategies can vary among populations of a single species across its range; in some species, the contribution can be approximately equal, while in others, one strategy can dominate in a specific area and time (Whitaker 2006, Starger et al. 2010).

During the life history of these long-lived organisms, sexual reproduction produces opportunities for generating new genetic combinations and opens possibilities for the dispersion of gametes and larvae within and between coral reefs or the colonization of new locations (van Oppen and Gates 2006). Asexual reproduction enables well-adapted local genotypes to increase the possibility of surviving disturbances and die-off events and expanding to nearby localities (Highsmith 1982, Baums et al. 2006). Asexual reproduction by fragmentation is advantageous because it provides a means of reaching sites where larvae are unable to settle (such as sandy areas at the periphery of a reef), it facilitates local dispersion and rapid occupation of space for the persistence of populations when conditions are unfavorable for sexual reproduction or larval recruitment, and it reduces the risk of genetic extinction (Highsmith 1982).

Branching corals are particularly susceptible to fragmentation from strong waves caused by hurricanes. Some corals appear to use fragmentation as part of their life histories after reaching a certain size (Highsmith 1982); however, fragmentation, as a reproductive strategy, is not exclusive to branched species. Long-lived massive corals, although less affected by storms, can be overturned or fragmented during these events (Lirman et al. 2001), and persist in local populations (Boulay et al. 2012). Genetic studies show that fragmentation contributes up to $25 \%$ of massive coral species (Miller and Ayre 2008). Thus, the evidence indicates that massive corals can propagate by asexual means, even though sexual strategies predominate (Miller and Ayre 2008, Boulay et al. 2012). To date, few massive coral species have been studied and no comparison has been made between species with different morphologies from the same sites.

Traditionally, the success of sexual reproduction has been addressed by following the presence or release of gametes and settlement of recruits (Harrison 2011, Schmidt-Roach et al. 2012), while the success of asexual reproduction by fragmentation has been addressed by counting fragments before and after disturbances and following the condition of naturally- or artificially-generated fragments (Smith and Hughes 1999). Genetic markers can be used to determine the genotypic diversity of a population, the number of distinct genets (genetically-different colonies originated by sexual means) or ramets (colonies generated by fragmantaion), giving the contribution of the reproductive strategy (sexual $v s$ asexual reproduction) to the existing genetic structure of the populations (Aranceta-Garza et al. 2012, Pinzón et al. 2012).

After the 1997-1998 El Niño event, coral reefs along the west coast of mainland Mexico experienced massive die-offs, with losses of 60-90\%; in the Gulf of California, die-offs were less than 18\% (Reyes-Bonilla et al. 2002). Despite aquellos causados por el hombre (Harrison 2011). La contribución de las estrategias de reproducción sexual y asexual puede variar entre las poblaciones de una misma especie a lo largo de su distribución; en algunas especies, la contribución puede ser aproximadamente igual, mientras que en otras, una estrategia puede dominar en un área y tiempo específico (Whitaker 2006, Starger et al. 2010).

Durante la historia de vida de estos organismos longevos, la reproducción sexual permite generar nuevas combinaciones genéticas, dispersar los gametos y las larvas dentro y entre los arrecifes coralinos o colonizar nuevas localidades (van Oppen y Gates 2006). La reproducción asexual permite que los genotipos bien adaptados localmente incrementen la posibilidad de sobrevivir a disturbios y eventos de mortalidad, y puedan expandirse a localidades cercanas (Highsmith 1982, Baums et al. 2006). La reproducción asexual por fragmentación tiene ventajas porque provee un medio para alcanzar sitios donde las larvas son incapaces de asentarse (como zonas arenosas en la periferia de un arrecife), facilita una dispersión local y una rápida posesión de espacio para la persistencia de poblaciones cuando las condiciones son desfavorables para la reproducción sexual o el reclutamiento de larvas, y reduce el riesgo de la extinción genética (Highsmith 1982).

Los corales ramificados son particularmente susceptibles a fragmentarse debido al fuerte oleaje causado por los huracanes. Algunos corales parecen utilizar la fragmentación como parte de su historia de vida después de haber alcanzado cierto tamaño (Highsmith 1982); sin embargo, la fragmentación, como una estrategia reproductiva, no es exclusiva de las especies de corales ramificados. Los corales masivos de larga vida, a pesar de ser menos afectados por las tormentas, pueden ser volteados o fragmentados durante estos eventos (Lirman et al. 2001) y persistir en las poblaciones locales (Boulay et al. 2012). Estudios genéticos demuestran que la fragmentación contribuye hasta en un 25\% de las especies de coral masivo (Miller y Ayre 2008). Esta evidencia indica que los corales masivos pueden propagarse por vía asexual, incluso aunque haya una predominancia de la estrategia de reproducción sexual (Miller y Ayre 2008, Boulay et al. 2012). A la fecha, se han estudiado pocas especies de coral masivo y no se ha realizado ninguna comparación entre especies con diferentes morfologías que se distribuyan en los mismos sitios.

Tradicionalmente, el éxito de la reproducción sexual de los corales se ha abordado mediante el seguimiento de la presencia o liberación de gametos y del asentamiento de reclutas (Harrison 2011, Schmidt-Roach et al. 2012), mientras que el éxito de la reproducción asexual por fragmentación ha sido abordado por medio del conteo de fragmentos antes y después de disturbios, y por el seguimiento de la condición de los fragmentos generados natural o artificialmente (Smith y Hughes 1999). Los marcadores genéticos pueden ser utilizados para determinar la diversidad genotípica de una población, el número de diferentes genets (colonias genéticamente 
massive die-offs, coral reefs along the west coast of Mexico recovered by sexual reproduction, as suggested in histological (Chávez-Romo and Reyes-Bonilla 2007, Carpizo-Ituarte et al. 2011, Rodríguez-Troncoso et al. 2011) and recruitment studies (Medina-Rosas et al. 2005, López-Pérez et al. 2007). However, to understand the function of coral communities in reef recovery following disturbances, it is necessary to disentangle the importance of reproductive strategies in the genetic structure of the populations.

In this study, two scleractinian species, Pocillopora damicornis and Porites panamensis, were selected because they are abundant and widespread on the west coast of Mexico, display contrasting morphology, and have different reproductive strategies (Chávez-Romo and Reyes-Bonilla 2007, Carpizo-Ituarte et al. 2011). Pocillopora damicornis is a branching and hermaphroditic species that reproduces sexually in the Gulf of California and Gulf of Panama by broadcast spawning (Glynn et al. 1991, Chávez-Romo and ReyesBonilla 2007), and is typically susceptible to fragmentation (fig. 1a). In contrast, P. panamensis is a massive and gonochoric species, sexually active along the west coast of Mexico and Panama (Glynn et al. 1994, Carpizo-Ituarte et al. 2011, Rodríguez-Troncoso et al. 2011). Asexual reproduction in massive corals is rare, but abrasion of whole colonies and multiple fragmentations of $P$. panamensis have been observed in the Gulf of California (fig. 1b). From this unusual situation, we explored the relative contribution of sexual and asexual reproductive strategies of $P$. damicornis and $P$. panamensis to the existing genetic structure of populations along the west coast of Mexico.

\section{MATERIALS AND METHODS}

\section{Locations and collection}

Collections were made in the most important regions of coral development off the west coast of mainland Mexico (Gulf of California, Bahía de Banderas, and Bahías de Huatulco; fig. 2). In the Gulf of California, samples of P. damicornis were collected at El Portugués (POR) and in the southern part of Bahía de La Paz (BLP); samples of P. panamensis were collected at Bahía de los Ángeles (BLA), Bahía Concepción (BCO), and BLP. Samples of both species were collected at Punta Arena de la Ventana (PAV), close to the entrance of the Gulf of California, and further south, at Isla Redonda (IRD) in Bahía de Banderas. In Bahías de Huatulco, P. damicornis was collected at Dos Hermanas (DOH) and La Entrega (LET); P. panamensis was collected only at LET. All coral samples were frozen in liquid nitrogen, transported to the laboratory, and stored at $-80{ }^{\circ} \mathrm{C}$.

\section{Allozyme electrophoresis}

Extractions of coral tissue were conducted in Stoddart's modified buffer (Stoddart 1983, Weil 1992) with a sonic distintas originadas por vía sexual) o ramets (colonias generadas por fragmentación), dando la contribución de una estrategia reproductiva (reproducción sexual vs reproducción asexual) a la estructura genética existente de las poblaciones (Aranceta-Garza et al. 2012, Pinzón et al. 2012).

Después del fenómeno de El Niño 1997-1998, los arrecifes coralinos a lo largo de la costa occidental de México experimentaron mortalidades masivas, con pérdidas de 60-90\%; en el golfo de California, las mortalidades fueron menores que el 18\% (Reyes-Bonilla et al. 2002). A pesar de las mortalidades masivas, los arrecifes de coral de la costa occidental de México se han recuperado por reproducción sexual, como se ha sugerido en estudios histológicos (Chávez-Romo y Reyes-Bonilla 2007, Carpizo-Ituarte et al. 2011, Rodríguez-Troncoso et al. 2011) y de reclutamiento (Medina-Rosas et al. 2005, López-Pérez et al. 2007). Sin embargo, para comprender la función que poseen las comunidades coralinas en la recuperación de los arrecifes después de disturbios, es necesario discernir la importancia de las estrategias reproductivas en la estructura genética de las poblaciones.

En este estudio, dos especies de corales escleractinios, Pocillopora damicornis y Porites panamensis, fueron seleccionados debido a que son muy abundantes y tienen una amplia distribución a lo largo de las costa occidental de México, exhiben un contraste en su morfología y poseen diferentes estrategias reproductivas (Chávez-Romo y ReyesBonilla 2007, Carpizo-Ituarte et al. 2011). Pocillopora damicornis es una especie ramificada y hermafrodita que se reproduce sexualmente en el golfo de California y el golfo de Panamá por liberación de gametos (Glynn et al. 1991, Chávez-Romo y Reyes-Bonilla 2007), y es comúnmente susceptible a la fragmentación (fig. 1a). En contraste, $P$. panamensis es una especie masiva y gonocórica, sexualmente activa a lo largo de toda la costa occidental de México y Panamá (Glynn et al. 1994, Carpizo-Ituarte et al. 2011, Rodríguez-Troncoso et al. 2011). La reproducción asexual en corales masivos es poco común, pero la abrasión de colonias completas y la generación múltiple de fragmentos de $P$. panamensis han sido observados en el golfo de California (fig. 1b). A partir de esta situación inusual, nosotros exploramos la contribución relativa de las estrategias reproductivas sexual y asexual de $P$. damicornis y $P$. panamensis a la existente estructura genética de las poblaciones de la costa occidental de México.

\section{MATERIALES Y MÉTODOS}

\section{Localidades y recolecta}

Las recolectas se realizaron en las regiones de desarrollo coralino más importantes a lo largo de la costa occidental de México (golfo de California, bahía de Banderas y bahías de Huatulco; fig. 2). En el golfo de California, se recolectaron muestras de $P$. damicornis en El Portugués (POR) y en el 

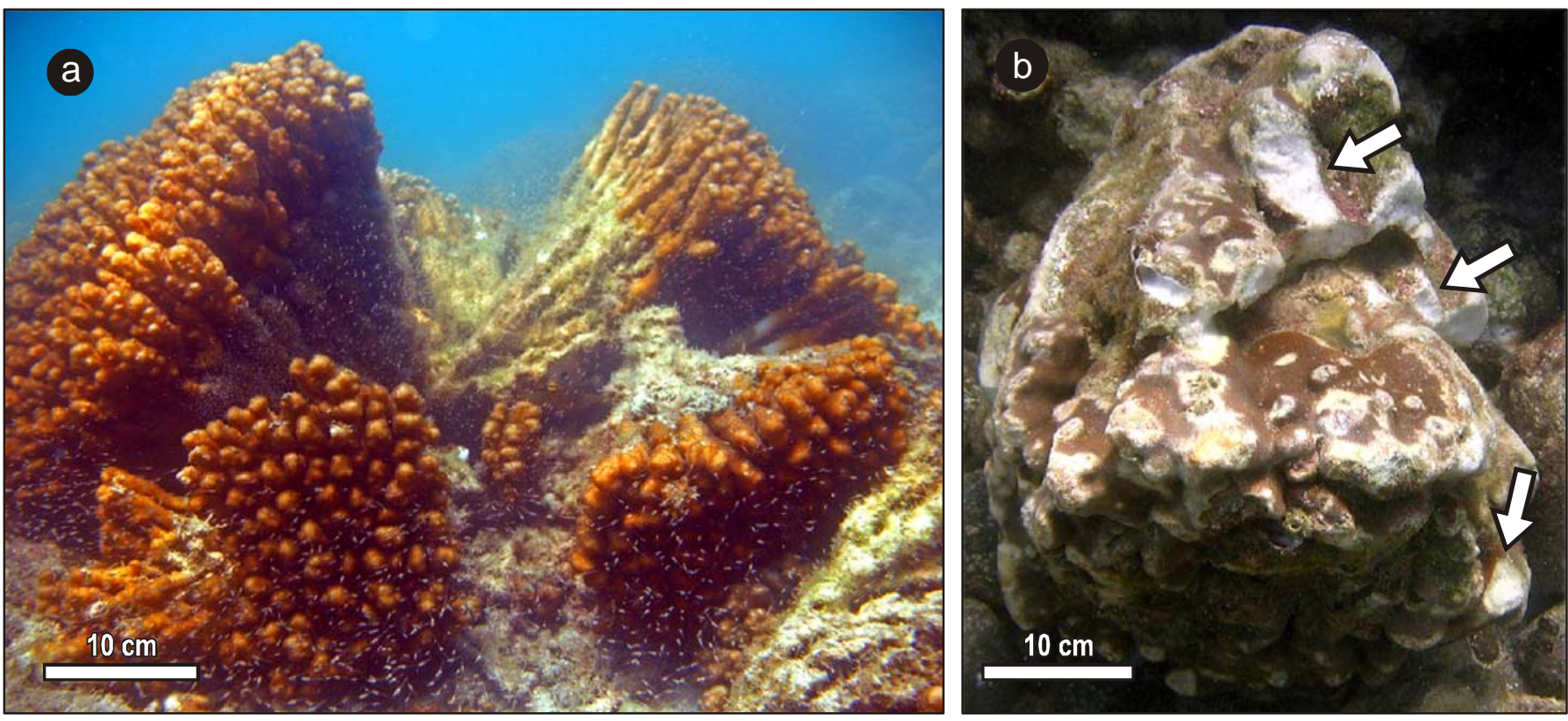

Figure 1. Fragmentation in (a) Pocillopora damicornis and (b) Porites panamensis in the Gulf of California, Mexico. Note multiple fragmentations in the $P$. panamensis colony, marked with arrows.

Figura 1. Fragmentación en (a) Pocillopora damicornis y (b) Porites panamensis en el golfo de California, México. Note las múltiples fragmentaciones de la colonia de $P$. panamensis, marcadas con flechas.

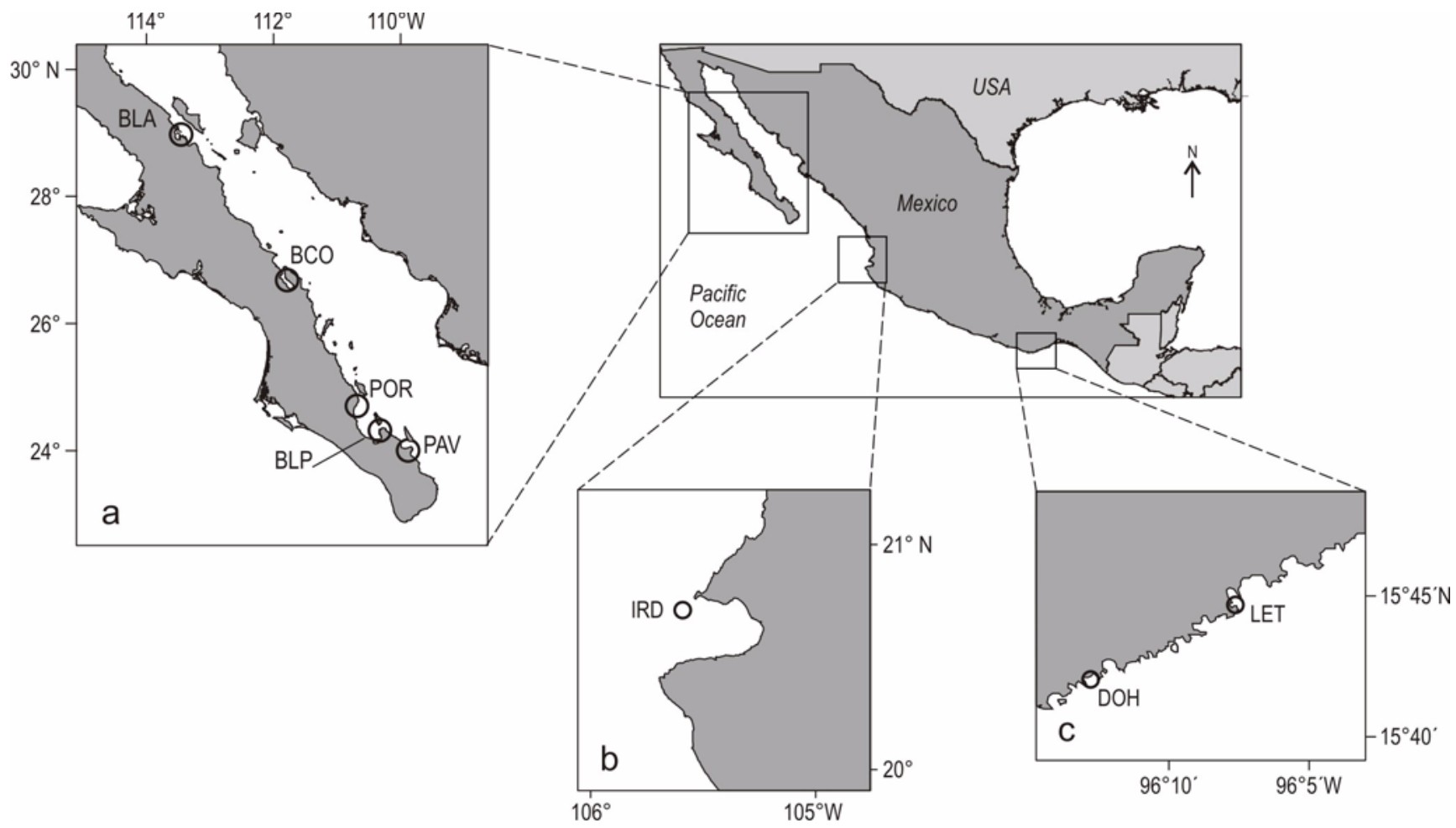

Figure 2. Regions and their respective locations sampled in this study. (a) Gulf of California: BLA, Bahía de los Ángeles; BCO, Bahía Concepción; POR, El Portugués; BLP, southern part of Bahía de La Paz; PAV, Punta Arena de la Ventana. (b) Bahía de Banderas: IRD, Isla Redonda. (c) Bahías de Huatulco: DOH, Dos Hermanas; LET, La Entrega.

Figura 2. Regiones y sus respectivas localidades muestreadas en el presente estudio. (a) Golfo de California: BLA, bahía de los Ángeles; BCO, bahía Concepción; POR, El Portugués; BLP, extremo sur de la bahía de La Paz; PAV, punta Arena de la Ventana. (b) Bahía de Banderas: IRD, Isla Redonda. (c) Bahías de Huatulco: DOH, Dos Hermanas; LET, La Entrega. 
dismembrator (model 100, Fisher Scientific, Waltham, MA). Then, $2 \mathrm{~mL}$ of the blastate was centrifuged at $2600 \times \mathrm{g}$ for $10 \mathrm{~min}$ at $4{ }^{\circ} \mathrm{C}$. The resulting supernatant was placed in vials and stored at $-80^{\circ} \mathrm{C}$ until analysis. The samples were analyzed after protein separation by polyacrylamide gel electrophoresis at $4{ }^{\circ} \mathrm{C}$ by a discontinuous gel system under native conditions (Manchenko 1994) using 8\% acrylamide. The multi-genotype of each colony was determined in four enzyme systems (five polymorphic loci) in both species (Paz-García et al. 2008b, Chávez-Romo et al. 2009). These enzyme systems were leucine-glycyl-glycyl peptidase (LGG-1, E.C. 3.4.11.1), malic enzyme (ME-1, E.C. 1.1.1.40), glutamate dehydrogenase (GDH-1 and $G D H-2$, E.C. 1.4.1.3), and esterase (EST-1 and EST-2, E.C. 3.1.1.1). Two loci were observed in the EST and GDH enzyme systems in P. damicornis and P. panamensis, respectively. Alleles at each locus were labeled alphabetically, based on decreasing mobility from the origin.

\section{Reproductive strategies}

We followed different approaches to estimate the contribution of sexual and asexual reproduction to the genetic structure. First, samples that had identical alleles at all five loci were identified as clone mates belonging to the same genet. Then, we obtained the number of unique multigenotypes detected at each location $\left(N_{g}\right)$ for each species. The probability of identity $\left(\mathrm{P}_{\mathrm{ID}}\right)$ was calculated to give a conservative estimate of the probability that two individuals sampled in the same general location share a multi-locus genotype by chance, not by descent (Waits et al. 2001). An overall unbiased $P_{\text {ID }}$ was calculated after sequentially multiplying $\mathrm{P}_{\mathrm{ID}}$ values for all loci, using the GIMLET software (Valière 2002); low overall $\mathrm{P}_{\mathrm{ID}}$ values for $P$. damicornis $\left(\mathrm{P}_{\mathrm{ID}}=2.9 \times 10^{-3}\right)$ and $P$. panamensis $\left(\mathrm{P}_{\mathrm{ID}}=0.88 \times 10^{-3}\right)$ indicated that the loci used in this study were accurate to assign ramets to clones.

Second, we calculated three reproductive indexes: (a) genotype richness $\left(N_{g}: N\right)$, where $N_{g}$ was calculated as described above and $N$ is the number of individuals analyzed at a location; (b) genotypic diversity $\left(G_{o}: G_{e}\right)$, where $G_{o}$ is the observed genotype diversity and $G_{e}$ is the expected genotype diversity; and (c) genotypic evenness $\left(G_{o}: N_{g}\right)$. Values close to one in the $N_{g}: N$ and $G_{o}: G_{e}$ ratios indicate that sexual reproduction is dominant at the location; values close to zero indicate locations that have colonies with high levels of asexual reproduction (Stoddart and Taylor 1988, Ayre et al. 1997). The $G_{o}: G_{e}$ ratio was calculated using the equations of Stoddart and Taylor (1988). Values close to one in the $G_{o}: N_{g}$ ratio indicate locations where each genet is represented by equal numbers of ramets; values close to zero indicate locations with one or few dominant clones (Coffroth and Lasker 1998). extremo sur de la bahía de La Paz (BLP); se recolectaron muestras de $P$. panamensis en bahía de Los Ángeles (BLA), bahía Concepción (BCO) y BLP. Se recolectaron muestras de las dos especies en punta Arena de la Ventana (PAV), cerca de la entrada del golfo de California, y hacia el sur, frente a isla Redonda (IRD) en bahía de Banderas. En bahías de Huatulco, P. damicornis fue recolectada en Dos Hermanas (DOH) y en La Entrega (LET); P. panamenis fue recolectada únicamente en LET. Todas las muestras de corales fueron congeladas en Nitrógeno líquido, transportadas al laboratorio y almacenadas a $-80^{\circ} \mathrm{C}$.

\section{Electroforesis de Aloenzimas}

Las extracciones de tejido de coral fueron realizadas en un tampón modificado de Stoddart (Stoddart 1983, Weil 1992) con un dismembrador sónico (modelo 100, Fisher Scientific, Waltham, MA). Posteriormente, se centrifugaron $2 \mathrm{~mL}$ del blastato a $2600 \times \mathrm{g}$ durante $10 \mathrm{~min}$ a $4{ }^{\circ} \mathrm{C}$. El sobrenadante resultante se colocó en viales y éstos se almacenaron a $-80{ }^{\circ} \mathrm{C}$ hasta el análisis. Las muestras fueron analizadas después de la separación de las proteínas por medio de electroforesis en gel de poliacrilamida a $4{ }^{\circ} \mathrm{C}$ por un sistema de gel de acrilamida al $8 \%$ discontinuo en condiciones nativas (Manchenko 1994). El multi-genotipo de cada colonia se determinó por medio de cuatro sistemas enzimáticos (cinco loci polimórficos) en ambas especies (Paz-García et al. 2008b, Chávez-Romo et al. 2009). Estos sistemas enzimáticos fueron leucina-glicil-glicil peptidasa ( $L G G-1$, E.C.3.4.11.1), enzima málica (ME-1, E.C1.1.1.40), glutamato deshidrogenasa (GDH-1 y $G D H-2$, E.C.1.4.1.3) y esterasa (EST-1 y EST-2, E.C 3.1.1.1). Se observaron dos loci en los sistemas enzimáticos EST y GDH en $P$. damicornis y P. panamensis, respectivamente. Los alelos en cada locus fueron designados alfabéticamente, con base en la diferencia de movilidad desde el origen.

\section{Estrategias reproductivas}

Se siguieron diferentes métodos para estimar la contribución de la reproducción sexual y asexual a la estructura genética. Primero, las muestras que presentaron alelos idénticos en los cinco loci fueron identificadas como clones pertenecientes a un mismo genet. Posteriormente, se obtuvo el número de multi-genotipos únicos detectados en cada localidad $\left(N_{g}\right)$ para cada especie. La probabilidad de identidad $\left(\mathrm{P}_{\text {ID }}\right)$ fue calculada para dar una estimación conservadora de la probabilidad de que dos individuos muestreados en la misma localidad compartieran un genotipo multi-locus por probabilidad, y no por descendencia (Waits et al. 2001). Se calculó un $\mathrm{P}_{\mathrm{ID}}$ global sin sesgo después de multiplicar secuencialmente los valores de $\mathrm{P}_{\mathrm{ID}}$ para todos los loci, utilizando el programa GIMLET (Valière 2002); los valores globales bajos de $\mathrm{P}_{\mathrm{ID}}$ para $P$. damicornis $\left(\mathrm{P}_{\mathrm{ID}}=2.9 \times 10^{-3}\right) \mathrm{y}$ 


\section{Cloning and frequency of disturbance}

To test whether there is a relationship between the level of cloning and frequency of disturbance, we tabulated the number of hurricanes that occurred at each location from 1958 through 2006. Standard buffer zones around each location were defined according to storm strength: $35 \mathrm{~km}$ for category 1 and 2 hurricanes (maximum sustained winds of $120-176 \mathrm{~km} / \mathrm{h}$ ), $60 \mathrm{~km}$ for category 3 hurricanes (177$212 \mathrm{~km} / \mathrm{h}$ ), and $100 \mathrm{~km}$ for category 4 and 5 hurricanes (>213 km/h) (Stoddart et al. 1985, Done 1992, Gardner et al. 2005). Data on occurrence and strength of hurricanes were obtained from the NOAA Historical Hurricane Tracks website (http://csc.noaa.gov/hurricanes/). Using this data set, a storm was considered if it entered a strength-specific buffer zone. Linear regressions were performed, where the number of storms approaching each location was the independent variable and the $N_{g}: N, G_{o}: G_{e}$, and $G_{o}: N_{g}$ ratios were the dependent variables.

\section{RESULTS AND DISCUSSION}

\section{Reproductive strategies of Pocillopora damicornis}

Two hundred $P$. damicornis colonies were sampled and most colonies showed unique genotypes $\left(N_{g}=136\right)$. The average percentage of unique multi-genotypes $\left(N_{g}\right)$ was 66.66 ( $\pm 16.45, S D)$, and the values at each location ranged from $35 \%$ to $83 \%$ (fig. 3). The $N_{g}: N$ average was 0.67 ( \pm 0.16 ), ranging from 0.35 to 0.83 (table 1 ); observed and expected genotypic diversity $\left(G_{o}: G_{e}\right)$ averaged $0.67 \pm 0.16$, ranging from 0.33 to 0.82 . The percentage of unique genotypes and reproductive indexes $\left(N_{g}: N\right.$ and $\left.G_{o}: G_{e}\right)$ indicate that $P$. damicornis uses sexual and asexual strategies. The $G_{o}: N_{g}$ ratio indicated that each genet is represented by almost equal numbers of ramets $(0.72 \pm 0.20)$, with values ranging from 0.31 to 0.84 (table 1 ). This result indicates that most of the locations are dominated by a few clones (i.e., high sexual reproduction indicated by $G_{\mathrm{o}}: N_{\mathrm{g}}$ values close to one), whereas in DOH there is high asexual reproduction (Coffroth and Lasker 1998).

The highest numbers of clonal colonies (42-56\%) were found in Bahías de Huatulco (DOH and LET), supporting earlier findings in the region. Previous histological studies based on surveys in Bahía de Banderas and Bahías de Huatulco over several years (2001-2004) did not detect mature gametes (only stages I-III), suggesting the predominance of asexual reproduction in these areas (Carpizo-Ituarte et al. 2011, Rodríguez-Troncoso et al. 2011). Interestingly, P. damicornis in Bahías de Huatulco had years of reproductive inactivity followed by periods of reproduction in subsequent years, but the causes for this have not yet been determined (Rodríguez-Troncoso et al. 2011).

Genetic studies of Pocillopora corals have shown evidence of asexual reproduction by fragmentation or by asexual
P. $\left(\mathrm{P}_{\mathrm{ID}}=0.88 \times 10^{-3}\right)$ indicaron que los loci utilizados en este estudio fueron precisos para asignar los ramets a los clones.

En segundo lugar, se calcularon tres índices reproductivos: (a) riqueza genotípica $\left(N_{g}: N\right)$, donde $N_{g}$ fue calculado como se decribe anteriormente y $N$ es el número de individuos analizados en una localidad; (b) diversidad genotípica $\left(G_{o}: G_{e}\right)$, donde $G_{o}$ es la diversidad del genotipo observada y $G_{e}$ es la diversidad del genotipo esperada; y (c) uniformidad genotípica $\left(G_{o}: N_{g}\right)$. Los valores cercanos a uno de los índices $N_{g}: N$ y $G_{o}: G_{e}$ indican que la reproducción sexual es dominante en la localidad; los valores cercanos a cero pertenecen a localidades con colonias con altos niveles de reproducción asexual (Stoddart y Taylor 1988, Ayre et al. 1997). El índice $G_{o}: G_{e}$ fue calculado con las ecuaciones de Stoddart y Taylor (1988). Los valores cercanos a uno del índice $G_{o}: N_{g}$ corresponden a localidades donde cada genet está representado por el mismo número de ramets; los valores cercanos a cero indican localidades con uno o pocos clones dominantes (Coffroth y Lasker 1998).

\section{Clonalidad y frecuencia de disturbios}

Para evaluar si hay una relación entre los niveles de clonación y la frecuencia de disturbios, se obtuvo el número de huracanes que incidieron en cada localidad desde 1958 hasta el 2006. Se definieron zonas estándar de amortiguamiento alrededor de cada sitio de acuerdo a la fuerza de la tormenta: $35 \mathrm{~km}$ para huracanes de categoría 1 y 2 (vientos sostenidos máximos de 120-176 km/h); $60 \mathrm{~km}$ para huracanes de categoría 3 (177-212 km/h); $100 \mathrm{~km}$ para huracanes de categoría 4 y 5 (>213 km/h) (Stoddart et al. 1985, Done 1992, Gardner et al. 2005). Los datos sobre la aparición y fuerza de los huracanes se obtuvieron en NOAA Historical Hurricane Tracks website (http://csc.noaa.gov/hurricanes/). Utilizando esta base de datos, una tormenta fue considerada solo si había entrado a la zona de amortiguamiento. Se realizaron regresiones lineales, donde la variable independiente fue el número de tormentas que se acercó a cada sitio y los razones $N_{g}: N, G_{o}: G_{e}$, y $G_{o}: N_{g}$ fueron las variables dependientes.

\section{RESULTADOS Y DISCUSIÓN}

\section{Estrategias reproductivas en Pocillopora damicornis}

Doscientas colonias de P. damicornis fueron recolectadas y la mayoría de las colonias mostraron genotipos únicos $\left(N_{g}=136\right)$. El porcentaje promedio de multi-genotipos únicos $\left(N_{g}\right)$ fue de $66.66( \pm 16.45$, desviación estándar) y los valores de cada sitio variaron de $35 \%$ a $83 \%$ (fig. 3). El promedio de $N_{g}: N$ fue de 0.67 ( \pm 0.16 ), y varió de 0.35 a 0.83 (tabla 1); la diversidad genotípica observada y esperada $\left(G_{o}: G_{e}\right)$ tuvo un promedio de $0.67 \pm 0.16$, y varió de 0.33 a 0.82 . El porcentaje de genotipos únicos e índices reproductivos $\left(N_{g}: N\right.$ y $\left.G_{o}: G_{e}\right)$ indicó que $P$. damicornis utiliza estrategias sexuales y asexuales. La proporción $G_{o}: N_{g}$ indicó que 


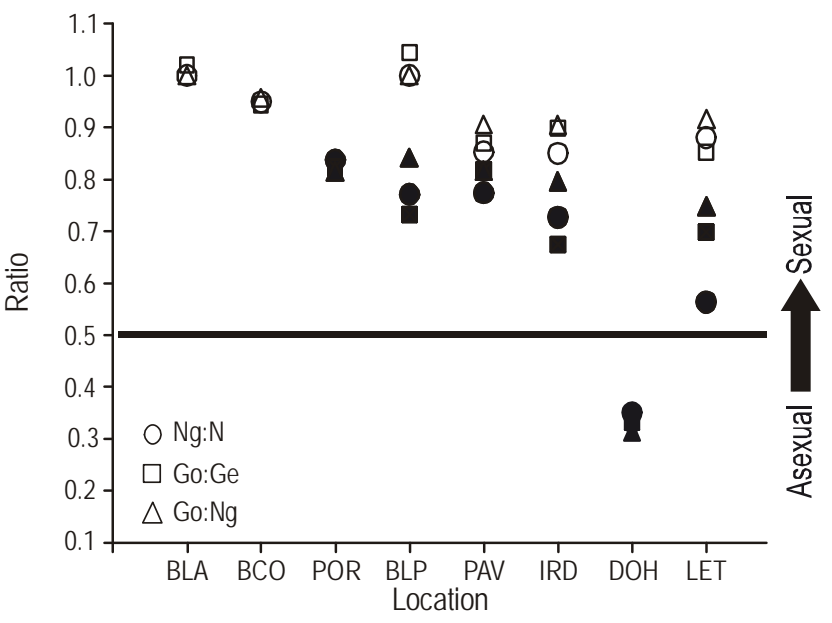

Figure 3. Relative contribution of sexual and asexual reproduction of Pocillopora damicornis (filled symbols) and Porites panamensis (open symbols) along the west coast of Mexico. $N_{g}: N$ and $G_{o}: G_{e}$ values close to one indicate dominance of sexual reproduction in the population, while lower values represent communities with some colonies formed by asexual reproduction. $G_{o}: N_{g}$ values close to one indicate populations of each genet represented by equal numbers of ramets, while values close to zero indicate populations with one or few dominant clones. $N$, number of individual colonies in each population; $N_{g}$, number of unique genotypes observed in each population; and $G_{o}$ and $G_{e}$, observed and expected genotypic diversity, respectively. Location abbreviations as in figure 2 .

Figura 3. Contribución relativa de la reproducción sexual y asexual de Pocillopora damicornis (símbolos rellenos) y Porites panamensis (símbolos en blanco) a lo largo de la costa occidental de México. Los valores cercanos a uno de los índices $N_{g}: N$ y $G_{o}: G_{e}$ indican dominancia de reproducción sexual en la población, mientras que los valores más bajos representan comunidades con algunas colonias formadas por reproducción asexual. Los valores cercanos a uno del índice $G_{o}: N_{g}$ indican poblaciones donde cada genet está representado por el mismo número de ramets, mientras que los valores cercanos a cero indican poblaciones con uno o varios clones dominantes. $N=$ número de colonias individuales en cada población, $N_{\mathrm{g}}=$ número de genotipos únicos observados en cada población, $G_{o}$ y $G_{e}=$ diversidad genotípica observada y esperada, respectivamente. Las abreviaciones de las localidades son como en la figura 2.

larvae in Japan (Adjeround and Tsuchiya 1999), Taiwan (Yeoh and Dai 2010), the west coast of Australia (Stoddart 1983), and the Gulf of California (Aranceta-Garza et al. 2012, Pinzón et al. 2012), indicating the tendency of this group to form clonal communities. The contribution of asexual reproduction to the coral communities is usually attributed to fragmentation caused by storms and hurricanes, low frequency of sexual reproduction, and disturbance (Highsmith 1982, Hunter 1993).

From 1958 through 2006, in the Gulf of California 44 hurricanes impacted the surrounding waters of these coral communities; 21 were category 1 and 2, 12 were category 3 , cada genet está representado por casi el mismo número de ramets $(0.72 \pm 0.20)$, con valores de 0.31 a 0.84 (tabla 1$)$. Este resultado indica que la mayoría de los sitios son dominados por unos pocos clones (i.e., alta reproducción sexual indicada por valores de $G_{o}: N_{g}$ cercanos a uno), mientras que en DOH hay una alta reproducción asexual (Coffroth y Lasker 1998).

El mayor número de colonias clonales (42-56\%) fue encontrado en las bahías de Huatulco (DOH y LET), lo cual confirma los hallazgos iniciales de la región. Los estudios histológicos previos realizados en bahía de Banderas y bahías de Huatulco durante varios años (2001-2004) no detectaron gametos maduros (solo estadios I-III), lo que sugiere la predominancia de reproducción asexual en estas áreas (CarpizoItuarte et al. 2011, Rodríguez-Troncoso et al. 2011). En bahías de Huatulco $P$. damicornis tuvo años de inactividad reproductiva seguida de periodos de reproducción en los años subsecuentes, pero las causas de esto aún no han sido determinadas (Rodríguez-Troncoso et al. 2011).

Estudios genéticos de corales Pocillopora han demostrado evidencia de reproducción asexual por fragmentación o por larvas asexuales en Japón (Adjeround y Tsuchiya 1999), Taiwan (Yeoh y Dai 2010), la costa occidental de Australia (Stoddart 1983) y el golfo de California (Aranceta-Garza et al. 2012, Pinzón et al. 2012), indicando la tendencia de este grupo a formar comunidades clonales. La contribución de la reproducción asexual a las comunidades de corales es usualmente atribuida a la fragmentación causada por tormentas y huracanes, a la poca frecuencia de reproducción sexual y a disturbios (Highsmith 1982, Hunter 1993).

Desde 1958 hasta 2006, en el golfo de California, 44 huracanes han impactado las aguas circundantes a estas comunidades coralinas; 21 huracanes pertenecieron a la categoría 1 y 2, 12 a la categoría 3, y 11 a la categorías 4 y 5 . Contrario a lo esperado, no se encontraron asociaciones significativas entre el índice reproductivo de $P$. damicornis y la frecuencia de huracanes a lo largo de la costa occidental de México (tabla 2). Pocos estudios han explorado la asociación de los huracanes y los índices reproductivos de corales con base en estudios genéticos (Baums et al. 2006, ArancetaGarza et al. 2012) o efectos de la reproducción en hábitats perturbados (Hunter 1993). Igual que los resultados del presente estudio, Baums et al. (2006) encontraron una estructura clonal del coral ramificado Acropora palmata en el Caribe, pero sin correlación con la incidencia de huracanes.

Aranceta-Garza et al. (2012) hallaron una relación significativa entre la frecuencia de huracanes y los índices de reproducción de Pocillopora verrucosa en el golfo de California (i.e., reproducción asexual por fragmentación). Las diferencias entre los resultados de su estudio y el presente pueden deberse a diversos factores. En primer lugar, $P$. verrucosa es más abundante que $P$. damicornis; incluso hay comunidades coralinas arrecifales que se componen casi en su totalidad por colonias de P. verrucosa (Aranceta-Garza et al. 2012, Paz-García et al. 2012a, Pinzón et al. 2012). Por 
Table 1. Reproductive strategy of Pocillopora damicornis along the west coast of Mexico. Location abbreviations as in figure 2. $N$, number of individuals analyzed at each location; $N_{g}$, number of unique multi-genotypes detected at each location; $N_{g}: N$, index of contribution of sexual reproduction; $G_{o}$, observed genotype diversity; $G_{e}$, is the expected genotype diversity; $G_{o}: G_{e}$, index of genotype diversity; $G_{o}: N_{g}$, index of genotypic evenness; $N_{\text {storm }}$, number of storms from 1958 through 2006.

Tabla 1. Estrategia reproductiva en Pocillopora damicornis a lo largo de la costa occidental de México. Abreviaciones de las localidades como en la figura 2. $N$, número de individuos analizados en el sitio; $N_{g}$, número de multi-genotipos únicos detectados en cada localidad; $N_{g}: N$, índice de contribución de la reproducción sexual; $G_{o}$, diversidad genotípica observada; $G_{e}$, diversidad genotípica esperada; $G_{o}: G_{e}$, índice de la diversidad genotípica; $G_{o}: N_{g}$, índice de uniformidad genotípica; $N_{\text {storm }}$, número de tormentas de 1958 hasta 2006.

\begin{tabular}{lccccccc}
\hline & \multicolumn{9}{c}{ Location } \\
\cline { 2 - 6 } & POR & BLP & PAV & IRD & DOH & LET & Mean (SD) \\
\hline$N$ & 43 & 48 & 31 & 22 & 40 & 16 & $33.33(11.45)$ \\
$N_{g}$ & 36 & 37 & 24 & 16 & 14 & 9 & $22.66(10.73)$ \\
$\% N_{g}$ & 83 & 77 & 77 & 72 & 35 & 56 & $66.66(16.45)$ \\
$N_{g}: N$ & 0.83 & 0.77 & 0.77 & 0.72 & 0.35 & 0.56 & $0.67(0.16)$ \\
$G_{o}$ & 29.34 & 31.13 & 19.61 & 12.73 & 4.39 & 6.73 & $17.33(10.33)$ \\
$G_{e}$ & 35.68 & 42.53 & 23.95 & 18.88 & 13.28 & 9.64 & $23.99(11.740)$ \\
$G_{o}: G_{e}$ & 0.82 & 0.73 & 0.81 & 0.67 & 0.33 & 0.69 & $0.67(0.16)$ \\
$G_{o}: N_{g}$ & 0.81 & 0.84 & 0.81 & 0.79 & 0.31 & 0.74 & $0.72(0.20)$ \\
$N_{\text {storm }}$ & 6 & 9 & 10 & 8 & 2 & 2 & $5.83(3.60)$ \\
\hline
\end{tabular}

and 11 were category 4 and 5 hurricanes. Contrary to expectations, no significant association was found between the $P$. damicornis reproductive index values and frequency of hurricanes along the west coast of Mexico (table 2). Few studies have explored the association of hurricanes and reproductive indexes in corals from genetic studies (Baums et al. 2006, Aranceta-Garza et al. 2012) or reproduction effects in disturbed habitats (Hunter 1993). Similar to the findings in this study, Baums et al. (2006) found higher clonal structure of the branching coral Acropora palmata in the Caribbean and no correlation with the incidence of hurricanes.

Aranceta-Garza et al. (2012) found a significant relationship between hurricane frequency and reproductive indexes of Pocillopora verrucosa in the Gulf of California (i.e., asexual reproduction by fragmentation). The differences in the results of their study and this study may be attributed to the following factors. First, $P$. verrucosa is more abundant than $P$. damicornis; in fact, some coral reef communities are composed almost entirely of $P$. verrucosa colonies (ArancetaGarza et al. 2012, Paz-García et al. 2012a, Pinzón et al. 2012). Therefore, this species is more susceptible to fragmentation than $P$. damicornis. Second, during storms, large pieces of reef framework can be broken, coral colonies can be overturned or transported, and branches can be fragmented into smaller sizes (Lirman et al. 2001). Survival of fragments after disturbances is related to their size (Smith and Hughes 1999); thus bigger fragments have a better chance of survival. As $P$. verrucosa has wider and longer branches than $P$. damicornis, it may be more successful at asexual reproduction by fragmentation and hence present an association with storms.
Table 2. Linear regressions between the reproductive indexes of Pocillopora damicornis and Porites panamensis and hurricane incidence on the west coast of Mexico.

Tabla 2. Regresiones lineales entre los índices reproductivos de Pocillopora damicornis y Porites panamensis y la incidencia de huracanes en la costa occidental de México.

\begin{tabular}{lccc}
\hline Species & Index & $r^{2}$ & $P$ \\
\hline P. damicornis & $N_{g}: N$ & 0.639 & 0.056 \\
& $G_{o}: G_{e}$ & 0.407 & 0.172 \\
& $G_{o}: N_{g}$ & 0.467 & 0.134 \\
P. panamensis & $N_{g}: N$ & 0.093 & 0.556 \\
& $G_{o}: G_{e}$ & 0.000 & 0.971 \\
& $G_{o}: N_{g}$ & 0.060 & 0.639 \\
\hline
\end{tabular}

lo tanto, esta especie es más susceptible a la fragmentación que $P$. damicornis. En segundo lugar, durante las tormentas, grandes piezas de la estructura del arrecife pueden fragmentarse, las colonias coralinas pueden ser volteadas o transportadas, y las ramas pueden ser fragmentadas en piezas de menor tamaño (Lirman et al. 2001). La supervivencia de los fragmentos después de los disturbios está relacionada con su tamaño (Smith y Hughes 1999); así los fragmentos más grandes tienen más posibilidades de sobrevivir. Como $P$. verrucosa posee ramas más gruesas y largas que $P$. damicornis, podría tener mayor éxito en la reproducción asexual por fragmentación y, por consiguiente, su índice reproductivo podría presentar una asociación con las tormentas. 
The highest number of colonies with unique genotypes (72-83\%) and the highest reproductive indexes in this study were found in the Gulf of California and Bahía de Banderas, suggesting that sexual reproduction plays a major role in these areas. These results are also supported by previous studies, which indicate higher recruitment levels of Pocillopora sp. in the Gulf of California (0.36 ind $\mathrm{m}^{-2} \mathrm{yr}^{-1}$, table 3), compared to the tropical part of the west coast of Mexico (López-Pérez et al. 2007, Cabral-Tena 2012; table 3). Moreover, it has been suggested that there was an exchange of propagules between coral communities after the 1997-1998 El Niño in the southern Gulf of California and Bahía de Banderas (Paz-García et al. 2012b). These findings indicate more favorable environmental conditions for sexual reproduction for $P$. damicornis and other coral species in the Gulf of California, which has an extended warm season and relatively more stable oceanographic conditions than Bahías de Huatulco (Chávez-Romo and Reyes-Bonilla 2007, Cabral-Tena 2012, Paz-García et al. 2012b). Thus, coral communities from the Gulf of California represent an important resource for generating genetic diversity by sexual
El mayor número de colonias con multi-genotipos únicos (72-83\%) e índices reproductivos más altos en este estudio fueron encontrados en el golfo de California y bahía de Banderas, lo que sugiere que la reproducción sexual tiene un papel más importante en estas áreas. Estos resultados están apoyados por estudios previos, los cuales indican altos niveles de reclutamiento de Pocillopora sp. en el golfo de California (0.36 ind $\mathrm{m}^{-2}$ año ${ }^{-1}$, tabla 3), en comparación con la parte tropical de la costa occidental de México (López-Pérez et al. 2007, Cabral-Tena 2012; tabla 3). Además, se ha sugerido la existencia de un intercambio de propágulos entre las comunidades coralinas del sur del golfo de California y bahía de Banderas después de El Niño 1997-98 (Paz-García et al. 2012b). Estos hallazgos indican condiciones ambientales más favorables para la reproducción sexual de $P$. damicornis y de otras especies de coral en el golfo de California, que cuenta con una estación cálida amplia y condiciones oceanográficas relativamente más estables que bahías de Huatulco (Chávez-Romo y Reyes-Bonilla 2007, Cabral-Tena 2012, Paz-García et al. 2012b). Por tanto, las comunidades coralinas del golfo de California representan un recurso importante

Table 3. Information on reproduction (histological studies), recruitment rate (estimated by tiles), and reproductive strategy inferred from genetic data of Pocillopora damicornis and Porites panamensis at three locations on the west coast of Mexico $\left(^{+}=0.58 \mathrm{yr}\right)$.

Tabla 3. Información de la reproducción (estudios histológicos), la tasa de reclutamiento (estimada por tejas) y la estrategia reproductiva inferida de los datos genéticos de Pocillopora damicornis y Porites panamensis en tres zonas de la costa occidental de México ${ }^{+}=0.58$ año).

\begin{tabular}{|c|c|c|c|}
\hline & $\begin{array}{c}\text { Bahía de La Paz } \\
\text { (Gulf of California) }\end{array}$ & $\begin{array}{c}\text { Isla Redonda } \\
\text { (Bahía de Banderas) }\end{array}$ & $\begin{array}{c}\text { La Entrega } \\
\text { (Bahías de Huatulco) }\end{array}$ \\
\hline \multicolumn{4}{|c|}{ Pocillopora damicornis (branching coral and broadcast spawner) } \\
\hline Gametes (stage) & I-IV & I-III & II-III \\
\hline Reproduction & $\begin{array}{c}\text { Sexual, } \\
\text { broadcast gametes (?) }\end{array}$ & $\begin{array}{c}\text { Asexual } \\
\text { fragmentation }\end{array}$ & $\begin{array}{c}\text { Asexual } \\
\text { fragmentation }\end{array}$ \\
\hline Recruitment rate (ind $\mathrm{m}^{-2} \mathrm{yr}^{-1}$ ) & 0.36 & $0^{+}$ & 0.21 \\
\hline Reproductive strategy & Sexual/asexual & Sexual/asexual & Asexual/sexual \\
\hline References* & $1,2,3$, this study & 4,5 , this study & 6,7 , this study \\
\hline
\end{tabular}

\section{Porites panamensis (Massive coral and brooder)}

Reproductive season

Oocytes per polyp

Reproductive potential

Range of number of recruits

Range of recruitment rate (ind $\mathrm{m}^{-2} \mathrm{yr}^{-1}$ )

Reproductive strategy

References*
Whole year

$$
\begin{gathered}
\text { 1-3 } \\
\text { High } \\
1-95
\end{gathered}
$$

0.71-78.71

Sexual

2, 8, 9, this study
Two months (August-September)

1

Low

$9^{+}$

1.08-4.66

Sexual

4, 5, 9, this study
Five months (May-September)

$1-2$

Moderate

4-96

$0.63-20.4$

Sexual

* References: 1 = Chávez-Romo and Reyes-Bonilla (2007); 2 = Cabral-Tena (2012); 3 = Pinzón et al. (2012); 4 = Medina-Rosas et al. (2005);

5 = Carpizo-Ituarte et al. (2011); 6 = Rodríguez-Troncoso et al. (2011); 7 = López-Pérez et al. (2007); 8 = Mora-Pérez (2005);

9 = Paz-García et al. (2008a). 
reproduction and exporting propagules for the recovery of damaged coral communities.

Pocillopora corals have been extensively studied, and the relative contribution of reproductive strategies has been inferred in different regions using genetic markers (Stoddart 1983, Adjeroud and Tsuchiya 1999, Miller and Ayre 2004, Sherman et al. 2006). In Japan, Taiwan, Australia, southwestern Mexico and the southern Gulf of California, asexual reproduction by fragmentation or the production of asexual larvae are the dominant reproductive strategy (Ward 1992, Adjeroud and Tsuchiya 1999, López-Pérez et al. 2007, Carpizo-Ituarte et al. 2011). In Indonesia, eastern Australia, and elsewhere in the Gulf of California, sexual reproduction is dominant (Sherman et al. 2006, Starger et al. 2010, Pinzón et al. 2012).

Additionally, histological studies have provided evidence that the reproductive strategy of $P$. damicornis varies (Glynn et al. 1991, Ward 1992, Chávez-Romo and Reyes-Bonilla 2007). Across its range, this species can spawn gametes for external fertilization or broadcast larvae (Stoddart 1983, Ayre and Miller 2004; see Harrison 2011 for a review). Shifts in modes of reproduction, from brooding larvae to free-spawning gametes, seem to occur in peripheral populations of Pocillopora off the coast of South Africa and the southwestern coast of Australia, and in the Red Sea and the Eastern Pacific (Glynn et al. 1991, Ward 1992, Chávez-Romo and Reyes-Bonilla 2007, Harrison 2011). However, different reproductive traits in $P$. damicornis across its distribution may be associated with cryptic species diversity (SchmidtRoach et al. 2012). Recent studies show that morphological plasticity is higher than previously thought (Flot et al. 2008, Souter 2010, Pinzón and LaJeunesse 2011). This will require detailed and integrated taxonomic studies (morphological and host-symbiont genetics) to unambiguously recognize Pocillopora species. In the studied locations, Pocillopora corals comprise one genetic group (Pocillopora type 1, sensu Pinzón and LaJeunesse 2011).

\section{Reproductive strategies of Porites panamensis}

We sampled 139 colonies of $P$. panamensis; most of the colonies had unique genotypes $\left(N_{g}=127\right)$. The unique multigenotypes ranged from $85 \%$ to $100 \%$ at each location (on average, $92.21 \pm 6.4$; fig. 3 ). The $N_{g}: N$ average was $0.92 \pm$ 0.06 , ranging from 0.85 to 1.00 among locations (table 4). Observed and expected genotypic diversity $\left(G_{o}: G_{e}\right.$ ratio) averaged $0.93 \pm 0.07$, ranging from 0.85 to 1.04 . The $G_{o}: N_{g}$ ratio revealed that, on average, each genet is represented by an equal number of ramets ( $0.94 \pm 0.04)$, ranging from 0.90 to 1.00 among locations (table 4); this result indicates high sexual reproduction, with $G_{o}: N_{g}$ values close to one (Coffroth and Lasker 1998). The percentage of unique genotypes and reproductive indexes showed that the massive coral $P$. panamensis mainly reproduced sexually along the west coast of Mexico, but also had low asexual reproduction at the para la generación de la diversidad genética mediante la reproducción sexual y la exportación de propágulos para la recuperación de comunidades coralinas dañadas.

Los corales Pocillopora han sido estudiados ampliamente, y la contribución relativa de estrategias reproductivas ha sido inferida en diferentes regiones utilizando marcadores genéticos (Stoddart 1983, Adjeroud y Tsuchiya 1999, Miller y Ayre 2004, Sherman et al. 2006). En Japón, Taiwan, Australia, el sur del golfo de California y en el suroeste de México, la reproducción asexual por fragmentación o la producción de larvas asexuales son las estrategias reproductivas dominantes (Ward 1992, Adjeroud y Tsuchiya 1999, LópezPérez et al. 2007, Carpizo-Ituarte et al. 2011). En Indonesia, el este de Australia y otras partes del golfo de California, la reproducción sexual es dominante (Sherman et al. 2006, Starger et al. 2010, Pinzón et al. 2012).

También, por medio de estudios histológicos se han presentado evidencias de la variación de las estrategias reproductivas en P. damicornis (Glynn et al. 1991, Ward 1992, Chávez-Romo y Reyes-Bonilla 2007). En toda su área de distribución, esta especie puede liberar gametos para la fertilización externa o expulsar larvas (Stoddart 1983, Ayre y Miller 2004, ver revisión en Harrison 2011). Parecen ocurrir cambios en los modos de reproducción, desde liberación de larvas incubadas a liberación de gametos, en las poblaciones periféricas de Pocillopora frente a la costa de Sudáfrica y la costa sudoccidental de Australia, y en el mar Rojo y Pacífico oriental (Glynn et al. 1991, Ward 1992, Chávez-Romo y Reyes-Bonilla 2007, Harrison 2011). Sin embargo, los diferentes aspectos reproductivos de $P$. damicornis a lo largo de su distribución pueden estar asociados a la diversidad de especies crípticas (Schmidt-Roach et al. 2012). Estudios recientes muestran que la plasticidad morfológica es mayor de lo que se pensaba previamente (Flot et al. 2008, Souter 2010, Pinzón y LaJeunesse 2011). Se necesitarían estudios taxonómicos detallados e integrales (morfológicos y genética hospedero-simbionte) para reconocer de forma inequívoca las especies de Pocillopora. En las localidades estudiadas, los corales Pocillopora comprenden un grupo genético (Pocillopora tipo 1, sensu Pinzón y LaJeunesse 2011).

\section{Estrategias reproductivas en Porites panamensis}

Se recolectaron 139 colonias de $P$. panamensis; la mayoría de las colonias tuvieron genotipos únicos $\left(N_{g}=127\right)$. Los multi-genotipos únicos variaron de $85 \%$ a $100 \%$ en cada sitio (en promedio, $92.21 \pm 6.4$; fig. 3 ). El promedio de $N_{g}: N$ fue de $0.92 \pm 0.06$, y varió de 0.85 a 1.00 entre sitios (tabla 4). La diversidad genotípica observada y esperada (índice $G_{o}: G_{e}$ ) tuvo un promedio de $0.93 \pm 0.07$, y varió de 0.85 a 1.04 . El índice $G_{o}: N_{g}$ indicó, en promedio, que cada genet está representado por un mismo número de ramets $(0.94 \pm 0.04)$, variando de 0.90 a 1.00 entre las localidades (tabla 4); este resultado indica una alta reproducción sexual, ya que los valores fueron cercanos a uno en el índice $G_{o}: N_{g}$ (Coffroth y 
Table 4. Reproductive strategies in Porites panamensis along the west coast of Mexico. Location abbreviations as in figure 2. $N$, number of individuals collected at each location; $N_{g}$, number of unique multi-genotypes detected at each location; $N_{g}: N$, index of contribution of sexual reproduction; $G_{o}$, observed genotype diversity; $G_{e}$, expected genotype diversity; $G_{o}: G_{e}$, index of genotype diversity; $G_{o}: N_{g}$, index of genotypic evenness; $N_{\text {storm }}$, number of storms from 1958 through 2006.

Tabla 4. Estrategias reproductivas en Porites panamensis a lo largo de la costa occidental de México. Las abreviaciones de las localidades son como en la figura 2. $N$, número de individuos analizados en el sitio; $N_{g}$, número de multi-genotipos únicos detectados en cada localidad; $N_{g}: N$, índice de contribución de la reproducción sexual; $G_{o}$, diversidad genotípica observada; $G_{e}$, diversidad genotípica esperada; $G_{o}: G_{e}$, índice de la diversidad genotípica; $G_{o}: N_{g}$, índice de uniformidad genotípica; $N_{\text {storm }}$, número de tormentas de 1958 hasta 2006.

\begin{tabular}{|c|c|c|c|c|c|c|c|}
\hline & \multicolumn{6}{|c|}{ Location } & Mean (SD) \\
\hline$N$ & 20 & 20 & 20 & 34 & 20 & 25 & $23.16(5.17)$ \\
\hline$N_{g}$ & 20 & 19 & 20 & 29 & 17 & 22 & $21.16(3.80)$ \\
\hline$\% N_{g}$ & 100 & 95 & 100 & 85 & 85 & 88 & $92.21(6.41)$ \\
\hline$G_{e}$ & $19.60(1.23)$ & 19.25 (1.65) & $19.13(1.76)$ & 30.18 (3.41) & $17.10(2.84)$ & 23.62 (2.19) & $21.48(4.35)$ \\
\hline$G_{o}: G_{e}$ & 1.02 & 0.94 & 1.04 & 0.87 & 0.90 & 0.85 & $0.93(0.07)$ \\
\hline$G_{o}: N_{g}$ & 1.00 & 0.95 & 1.00 & 0.90 & 0.90 & 0.91 & $0.94(0.04)$ \\
\hline$N_{\text {storm }}$ & 2 & 4 & 9 & 10 & 8 & 2 & $5.83(3.60)$ \\
\hline
\end{tabular}

southern locations. Recent studies of massive coral species show that fragmentation is higher than previously thought (Miller and Ayre 2008, Boulay et al. 2012). In this study, 24-30\% of $P$. panamensis colonies at PAV, IRD, and LET originated by asexual reproduction. Similar percentages of asexual reproduction (8-25\%) have been estimated for other four massive species: Porites lobata (Boulay et al. 2012), Montastraea annularis (Severance and Karl 2006), Montastraea faveolata (Severance and Karl 2006), and Platygyra daedalea (Miller and Ayre 2008).

In the Gulf of California, detached fragments and whole P. panamensis colonies were observed (fig. 1). No significant association was found between the reproductive index values and frequency of hurricanes along the west coast of Mexico for $P$. panamensis colonies (table 2). Different processes may contribute to fragmentation in this species. For example, bioerosion by polychaetes, bivalves, and excavating sponges could make the coral skeleton more susceptible to fragmentation (Carriquiry and Reyes-Bonilla 1997, ReyesBonilla 2003), or differences in regeneration and partial mortality may cause fission of large colonies (Paz-García and Reyes-Bonilla 2006).

Histological studies of $P$. panamensis indicate that successful sexual reproduction is feasible along the west coast of Mexico (table 3). Release of larvae occurs most of the year in BLP (Gulf of California), but usually during summer (two-four months) in Bahía de Banderas and Bahías de Huatulco (Carpizo-Ituarte et al. 2011, Rodríguez-Troncoso et al. 2011, Paz-García et al. 2008a; see table 3). Other differences along the west coast of Mexico are that the Gulf of California had the highest $N_{g}: N$ and $G_{o}: G_{e}$ ratios and number
Lasker 1998). El porcentaje de multi-genotipos únicos e índices reproductivos mostró que el coral masivo $P$. panamensis se reprodujo principalmente de forma sexual a lo largo de la costa occidental de México, pero también presentó una reproducción asexual en las localidades del sur. Estudios recientes de especies de corales masivos muestran que la fragmentación es mayor de lo que se pensaba anteriormente (Miller y Ayre 2008, Boulay et al. 2012). En el presente estudio, 24-30\% de las colonias de P. panamensis en PAV, IRD, y LET se originaron por reproducción asexual. Se han estimado porcentajes similares de reproducción asexual (8-25\%) para otras cuatro especies masivas: Porites lobata (Boulay et al. 2012), Montastraea annularis (Severance y Karl 2006), Montastraea faveolata (Severance y Karl 2006) y Platygyra daedalea (Miller y Ayre 2008).

En el golfo de California, se observaron fragmentos desprendidos y colonias completas de $P$. panamensis (fig. 1b). No se encontró una asociación significativa entre los valores del índice reproductivo de $P$. panamensis y la frecuencia de huracanes a lo largo de la costa occidental de México (tabla 2). Diferentes procesos podrían contribuir a la fragmentación de esta especie. Por ejemplo, la bioerosión por poliquetos, bivalvos y esponjas excavadoras podría provocar que el esqueleto sea más susceptible a la fragmentación (Carriquiry y Reyes-Bonilla 1997, Reyes-Bonilla 2003), o las diferencias en la regeneración y la mortalidad parcial podrían causar fisión en grandes colonias (Paz-García y Reyes-Bonilla 2006).

Algunos estudios histológicos de $P$. panamensis indican que la reproducción sexual exitosa es factible a lo largo de la costa occidental de México (tabla 3). La liberación de larvas 
of oocytes per polyp (Mora-Pérez 2005, Carpizo-Ituarte et al. 2011, Rodríguez-Troncoso et al. 2011), suggesting that even with relatively large changes in environmental conditions over an annual cycle, this coral is well adapted for survival and reproduces under harsh conditions.

High recruitment ratios of Porites in the Gulf of California and Bahías de Huatulco (0.63-78.71 ind $\mathrm{m}^{-2} \mathrm{yr}^{-1}$, see table 3) suggest recovery of coral reefs in other areas of the west coast of Mexico because their reproduction ratios are high (López-Pérez et al. 2007, Cabral-Tena 2012, PazGarcía et al. 2012b). Differences in reproductive strategies of coral communities in the Gulf of California could have evolutionary significance because there is high reproduction potential when there are multiple larval releases. This may be a survival strategy under fluctuating environmental conditions. Long-term studies and more attention to massive species are necessary to understand the role of asexual reproduction and population dynamics among coral reefs.

In summary, this study provides genetic evidence of the importance of sexual and asexual reproduction in the reef corals P. damicornis and P. panamensis at diverse sites along the west coast of Mexico. These results support previous histological and recruitment studies in this region. Our findings indicate that locations in the Gulf of California have high levels of sexual reproduction, recruitment, and genotypic diversity of corals that could be important for recovery in other locations (Saavedra-Sotelo et al. 2011, Paz-García et al. 2012b). Further integrated research of coral communities would improve our understanding of population structure and reproductive response to global climate change in these ecosystems.

\section{ACKNOWLEDGMENTS}

This study was funded by the Project AWARE Foundation (to DAPG) and SEMARNAT project 2002-c01-0605 (to Luis Calderon of CICESE). HECR (\#198888) and DAPG (\#160065) are recipients of fellowship grants from the National Council for Science and Technology (CONACYT, Mexico). We thank the work group at the Parque Nacional Islas Marietas, and Diana Sánchez, Luis Lombardo, and Karina Xolaltenco (UABC) for their laboratory assistance. Salwa El Khattabi (UABCS) helped with the Spanish translation of the paper. Ira Fogel (CIBNOR) provided editorial services.

\section{REFERENCES}

Adjeround M, Tsuchiya M. 1999. Genetic variation and clonal structure in the scleractinian coral Pocillopora damicornis in the Ryukyu Archipelago, southern Japan. Mar. Biol. 134: 753-769. http://dx.doi.org/10.1007/s002270050592

Aranceta-Garza F, Balart EF, Reyes-Bonilla H, Cruz-Hernández P. 2012. Effect of tropical storms on sexual and asexual reproduction in coral Pocillopora verrucosa subpopulations in the Gulf of California. Coral Reefs 31: 1157-1167. http://dx.doi.org/10.1007/s00338-012-0941-9 ocurre la mayor parte del año en BLP (golfo de California), pero usualmente durante el verano (de dos a cuatro meses) en bahía de Banderas y bahías de Huatulco (Carpizo-Ituarte et al. 2011, Rodríguez-Troncoso et al. 2011, Paz-García et al. 2008a; ver tabla 3). Otras diferencias a lo largo de la costa occidental de México son que en el golfo de California se presentaron los valores más altos de $N_{g}: N$ y $G_{o}: G_{e}$, y el mayor número de ovocitos por pólipo (Mora-Pérez 2005, CarpizoItuarte et al. 2011, Rodríguez-Troncoso et al. 2011), lo cual sugiere que incluso con grandes cambios en las condiciones ambientales durante un ciclo anual, este coral está bien adaptado para sobrevivir y reproducirse bajo condiciones ambientales drásticas.

Las altas tasas de reclutamiento de Porites en el golfo de California y bahías de Huatulco (0.63-78.71 ind $\mathrm{m}^{-2}$ año-1, ver tabla 3) sugieren la recuperación de los arrecifes coralinos en otras áreas de la costa occidental de México porque sus valores en los índices de reproducción son altos (LópezPérez et al. 2007, Cabral-Tena 2012, Paz-García et al. 2012b). Las diferencias en las estrategias reproductivas de las comunidades coralinas en el golfo de California podrían tener un significado evolutivo debido a que hay un alto potencial reproductivo cuando ocurren múltiples liberaciones de larvas al año. Este comportamiento puede ser una estrategia de supervivencia bajo condiciones ambientales fluctuantes. Son necesarios estudios a largo plazo y una mayor atención a especies masivas para poder comprender el rol de la reproducción asexual y la dinámica de poblaciones entre los arrecifes coralinos.

En resumen, este estudio provee evidencia genética de la importancia de la reproducción sexual y asexual en los corales arrecifales $P$. damicornis y $P$. panamensis en varias localidades a lo largo de la costa occidental de México. Estos resultados confirman los hallazgos previos de los estudios histológicos y de reclutamiento realizados en esta región. Nuestros resultados indican que las localidades en el golfo de California poseen altos niveles de reproducción sexual, reclutamiento y de diversidad genotípica que pudieran ser importantes para la recuperación de corales en otras localidades a lo largo de la costa occidental de México (SaavedraSotelo et al. 2011, Paz-García et al. 2012b). Futuras investigaciones integrales de las comunidades coralinas podrían mejorar nuestra comprensión de la estructura poblacional y respuesta reproductiva ante el cambio climático en estos ecosistemas.

\section{Agradecimientos}

Este proyecto fue financiado por la Fundación Project AWARE (proyecto a DAPG) y el proyecto SEMARNAT 2002-c01-0605 (a Luis Calderón del CICESE). HECR (\#198888) y DAPG (\#160065) obtuvieron becas por el Consejo Nacional de Ciencia y Tecnología. Agradecemos al grupo de trabajo del Parque Nacional Islas Marietas, y a 
Ayre DJ, Miller KJ. 2004. Where do clonal coral larvae go? Adult genotypic diversity conflicts with reproductive effort in the brooding coral Pocillopora damicornis. Mar. Ecol. Prog. Ser. 277: 95-105.

http://dx.doi.org/10.3354/meps277095

Ayre DJ, Hughes TP, Standish RJ. 1997. Genetic differentiation, reproductive mode, and gene flow in the brooding coral Pocillopora damicornis along the Great Barrier Reef, Australia. Mar. Ecol. Prog. Ser. 159: 175-187. http://dx.doi.org/10.3354/meps159175

Baums IB, Miller MW, Hellberg ME. 2006. Geographic variation in clonal structure in a reef-building Caribbean coral, Acropora palmata. Ecol. Monogr. 76: 503-519.

Boulay JN, Cortes J, Nivia-Ruiz J, Baums IB. 2012. High genotypic diversity of the reef-building coral Porites lobata (Scleractinia: Poritidae) in Isla del Coco National Park, Costa Rica. Rev. Biol. Trop. 60: 279-292.

Cabral-Tena RA. 2012. Reclutamiento coralino en un arrecife restaurado en La Paz, BCS. In: Pérez-Ramírez M, Lluch-Cota SE (eds.), Biodiversidad y Vulnerabilidad de Ecosistemas Costeros en Baja California Sur. Centro de Investigaciones Biológicas del Noroeste, La Paz, BCS, México, pp. 331-345.

Carpizo-Ituarte E, Vizcaíno-Ochoa V, Chi-Barragán G, TapiaVázquez O, Cupul-Magaña AL, Medina-Rosas P. 2011. Evidence of sexual reproduction in the hermatypic corals Pocillopora damicornis, Porites panamensis, and Pavona gigantea in Banderas Bay, Mexican Pacific. Cienc. Mar. 37: 97-112. http://dx.doi.org/10.7773/cm.v37i1.1773

Carriquiry JD, Reyes-Bonilla H. 1997. Estructura de la comunidad y distribución geográfica de los arrecifes coralinos de Nayarit, Pacífico de México. Cienc. Mar. 23: 227-248.

Chávez-Romo HE, Reyes-Bonilla H. 2007. Sexual reproduction of the coral Pocillopora damicornis in the southern Gulf of California, Mexico. Cienc. Mar. 33: 495-501.

Chávez-Romo HE, Correa-Sandoval F, Paz-García DA, ReyesBonilla H, López-Pérez RA, Medina-Rosas P, HernándezCortés MP. 2009. Genetic structure of a scleractinian coral, Pocillopora damicornis, in the Mexican Pacific. Proc. 11th Int. Coral Reef Symp., pp. 429-433.

Coffroth MA, Lasker HR. 1998. Population structure of a clonal gorgonian coral: The interplay between clonal reproduction and disturbance. Evolution 52: 379-393.

Done TJ. 1992. Effects of tropical cyclone waves on ecological and geomorphological structures on the Great Barrier Reef. Cont. Shelf Res. 12: 859-872.

Flot J-F, Magalo H, Cruaud C, Couloux A, Tillier S. 2008. Patterns of genetic structure among Hawaiian corals of the genus Pocillopora yield clusters of individuals that are compatible with morphology. C. R. Biol. 331: 239-247.

Gardner TA, Cote IM, Gill JA, Grant A, Watkinson AR. 2005. Hurricanes and Caribbean coral reefs: Impacts, recovery patterns, and role in long-term decline. Ecology 86: 174-184. http://dx.doi.org/10.1890/04-0141

Glynn PW, Gassman NJ, Eakin CM, Cortés J, Smith DB, Guzmán HM. 1991. Reef coral reproduction in the eastern Pacific: Costa Rica, Panama, and Galapagos Islands (Ecuador). I. Pocilloporidae. Mar. Biol. 109: 355-368. http://dx.doi.org/10.1007/BF01313501

Glynn PW, Colley SB, Eakin CM, Smith DB, Cortés J, Gassman NJ, Guzmán HM, Del Rosario JB, Feingold JS. 1994. Reef coral reproduction in the eastern Pacific: Costa Rica, Panama, and Galapagos Islands (Ecuador). II. Poritidae. Mar. Biol. 118: 191-208.

http://dx.doi.org/10.1007/BF00349785
Diana Sánchez, Luis Lombardo y Karina Xolaltenco (UABC) su asistencia en el laboratorio. Salwa El Khattabi (UABCS) ayudó en la traducción al español del artículo. Ira Fogel (CIBNOR) proporcionó servicios editoriales.

Harrison PL. 2011. Sexual reproduction of scleractinian corals. In: Dubinsky Z, Stambler N (eds.), Coral Reefs: An Ecosystem in Transition. Springer, New York, pp. 59-85.

Highsmith R. 1982. Reproduction by fragmentation in corals. Mar. Ecol. Prog. Ser. 7: 207-226.

Hunter CL. 1993. Clonal diversity and population structure of scleractinian coral populations under different disturbance histories. Evolution 47: 1213-1228.

Lirman D, Glynn PW, Baker AC, Leyte-Morales GE. 2001. Combined effects of three sequential storms on the Huatulco coral reef tract, Mexico. Bull. Mar. Sci. 69: 267-278.

López-Pérez RA, Mora-Pérez MG, Leyte-Morales GE. 2007. Coral (Anthozoa: Scleractinia) recruitment at Bahías de Huatulco, western Mexico: Implications for coral community structure and dynamics. Pac. Sci. 61: 355-369. http://dx.doi.org/10.1353/psc.2007.0032

Manchenko GP. 1994. Handbook of Detection of Enzymes on Electrophoretic Gels. CRC Press, Boca Raton, FL. 554 pp.

Medina-Rosas P, Carriquiry JD, Cupul-Magaña AL. 2005. Recruitment of Porites (Scleractinia) on artificial substrate in reefs affected by the 1997-98 El Niño in Banderas Bay, Mexican Pacific. Cienc. Mar. 31: 103-109. http://dx.doi.org/10.7773/cm.v31i11.75

Miller KJ, Ayre DJ. 2004. The role of sexual and asexual reproduction in structuring high latitude populations of the reef coral Pocillopora damicornis. Heredity 92: 557-568. http://dx.doi.org/10.1038/sj.hdy.6800459

Miller KJ, Ayre DJ. 2008. Population structure is not a simple function of reproductive mode and larval type: Insights from tropical corals. J. Anim. Ecol. 77: 713-724. http://dx.doi.org/10.1111/j.1365-2656.2008.01387.x

Mora-Pérez MG. 2005. Biología reproductiva del coral Porites panamensis Verrill 1866 (Anthozoa: Scleractinia), en Bahía de La Paz, Baja California Sur, México. MSc thesis, Centro Interdisciplinario de Ciencias Marinas, La Paz, BCS, México. $81 \mathrm{pp}$.

Paz-García DA, Reyes-Bonilla H. 2006. Temporal variation in the regeneration rate of artificial lesions in two morphotypes of Porites panamensis. Cienc. Mar. 32: 187-194.

Paz-García DA, Reyes-Bonilla H, Hernández-Cortés MP. 2008a. Genetic variation in two morphotypes of Porites panamensis from the Gulf of California, Mexico. Proc. 11th Int. Coral Reef Symp., pp. 444-448.

Paz-García DA, Correa-Sandoval F, Chávez-Romo HE, ReyesBonilla H, López-Pérez A, Medina-Rosas P, Hernández-Cortés MP. 2008b. Genetic structure of the massive coral Porites panamensis (Anthozoa: Scleractinia) from the Mexican Pacific. Proc. 11th Int. Coral Reef Symp., pp. 449-453.

Paz-García DA, Balart EF, García-de-León, FJ. 2012a. Cold water bleaching of Pocillopora in the Gulf of California. Proc. 12th Int. Coral Reef Symp. 9A-10: 1-5.

Paz-García DA, Chávez-Romo HE, Correa-Sandoval F, ReyesBonilla H, López-Pérez A, Medina-Rosas P, Hernández-Cortés MP. 2012b. Genetic connectivity patterns of corals Pocillopora damicornis and Porites panamensis (Anthozoa: Scleractinia) along the west coast of Mexico. Pacific Sci. 66: 43-61. http://dx.doi.org/10.2984/66.1.3 
Pinzón JH, LaJeunesse TC. 2011. Species delimitation of common reef corals in the genus Pocillopora using nucleotide sequence phylogenies, population genetics and symbiosis ecology. Mol. Ecol. 20: 311-325. http://dx.doi.org/10.1111/j.1365-294X.2010.04939.x

Pinzón JH, Reyes-Bonilla H, Baums IB, LaJeunesse TC. 2012. Contrasting clonal structure among Pocillopora (Scleractinia) communities at two environmentally distinct sites in the Gulf of California. Coral Reefs. 31: 765-777. http://dx.doi.org/10.1007/s00338-012-0887-y

Reyes-Bonilla H. 2003. Coral reefs of the Pacific coast of Mexico. In: Cortés J (ed.), Latin American Coral Reefs. Elsevier Science, Amsterdam, pp. 331-349.

Reyes-Bonilla H, Carriquiry JD, Leyte-Morales GE, Cupul-Magaña AL. 2002. Effects of the El Niño-Southern Oscillation and the anti-El Niño event (1997-1999) on coral reefs of the western coast of Mexico. Coral Reefs 21: 368-372. http://dx.doi.org/10.1007/s00338-002-0255-4

Rodríguez-Troncoso AP, Carpizo-Ituarte E, Leyte-Morales GE, ChiBarragán G, Tapia-Vázquez O. 2011. Sexual reproduction of three coral species from the Mexican South Pacific. Mar. Biol. 158: 2673-2683. http://dx.doi.org/10.1007/s00227-011-1765-9

Saavedra-Sotelo NC, Calderón-Aguilera LE, Reyes-Bonilla RA, López-Pérez RA, Medina-Rosas P, Rocha-Olivares A. 2011. Limited genetic connectivity of Pavona gigantea in the Mexican Pacific. Coral Reefs 30: 677-686. http://dx.doi.org/10.1007/s00338-011-0742-6

Schmidt-Roach S, Miller KJ, Woolsey E, Gerlach G, Baird AH. 2012. Broadcast spawning by Pocillopora species on the Great Barrier Reef. PLoS ONE 7(12): e50847. http://dx.doi.org/10.1371/journal.pone.0050847

Severance EG, Karl SA. 2006. Contrasting population genetic structure of sympatric mass-spawning Caribbean corals. Mar. Biol. 150: 57-68. http://dx.doi.org/10.1007/s00227-006-0332-2

Sherman CDH, Ayre DJ, Miller KJ. 2006. Asexual reproduction does not produce clonal populations of the brooding coral Pocillopora damicornis on the Great Barrier Reef, Australia. Coral Reefs 25: 7-18. http://dx.doi.org/10.1007/s00338-005-0053-x

Smith LD, Hughes TP. 1999. An experimental assessment of survival, re-attachment and fecundity of coral fragments. J. Exp.
Mar. Biol. Ecol. 235: 147-164.

http://dx.doi.org/10.1016/S0022-0981(98)00178-6

Souter P. 2010. Hidden genetic diversity in a key model species of coral. Mar. Biol. 157: 875-885.

http://dx.doi.org/10.1007/s00227-009-1370-3

Starger CJ, Barber PH, Ambariyanto Baker AC. 2010. The recovery of coral genetic diversity in the Sunda Strait following the 1883 eruption of Krakatau. Coral Reefs 29: 547-565. http://dx.doi.org/10.1007/s00338-010-0609-2

Stoddart JA. 1983. Asexual production of planulae in the coral Pocillopora damicornis. Mar. Biol. 76: 279-284. http://dx.doi.org/10.1007/BF00393029

Stoddart JA, Taylor JF. 1988. Genotypic diversity: Estimation and prediction in samples. Genetics 118: 705-711

Stoddart JA, Ayre DJ, Willis B, Heyward AJ. 1985. Self-recognition in sponges and corals. Evolution 39: 461-463.

Valière N. 2002. GIMLET: A computer program for analyzing genetic individual identification data. Mol. Ecol. Notes 2: 377-379. http://dx.doi.org/10.1046/j.1471-8286.2002.00228.x-i2

Van Oppen MJH, Gates RD. 2006. Conservation genetics and the resilience of reef-building corals. Mol. Ecol. 15: 3863-3883. http://dx.doi.org/10.1111/j.1365-294X.2006.03026.x

Waits LP, Luikart G, Taberlet P. 2001. Estimating the probability of identity among genotypes in natural populations: Cautions and guidelines. Mol. Ecol. 10: 249-256. http://dx.doi.org/10.1046/j.1365-294X.2001.01185.x

Ward S. 1992. Evidence for broadcast spawning as well as brooding in the scleractinian coral Pocillopora damicornis. Mar. Biol. 112: 641-646. http://dx.doi.org/10.1007/BF00346182

Weil E. 1992. Genetic and morphlogical variation in Caribbean and eastern Pacific Porites (Anthozoa, Scleractinia). Preliminary results. Proc. 7th Int. Coral Reef Symp. 2: 643-656.

Whitaker K. 2006. Genetic evidence for mixed modes of reproduction in the coral Pocillopora damicornis and its effect on population structure. Mar. Ecol. Prog. Ser. 306: 115-124. http://dx.doi.org/10.3354/meps306115

Yeoh S, Dai C. 2010. The production of sexual and asexual larvae within single broods of the scleractinian coral, Pocillopora damicornis. Mar. Biol. 157: 351-359. http://dx.doi.org/10.1007/s00227-009-1322-y

Received January 2013, received in revised form October 2013, accepted October 2013. 